\title{
Resposta de Cultivares de Algodoeiro a Subdoses de GLYPHOSATE $^{1}$
}

\author{
Response of Cotton Cultivars to Reduced Rates of Glyphosate
}

YAMASHITA, O.M. ${ }^{2}$ e GUIMARÃES, S.C. ${ }^{3}$

\begin{abstract}
RESUMO - Avaliou-se a resposta de nove cultivares de algodoeiro, de importância econômica no Estado do Mato Grosso, quanto à intoxicação causada por subdoses de glyphosate. Os cultivares de algodoeiro utilizados foram Fabrika, Makina, ITA-90, FM 986, FM 966, Delta Opal, BRS Facual, Antares e Coodetec 407. As plantas foram cultivadas em tubetes preenchidos com substrato de solo e mantidas em casa telada, tendo recebido a aplicação do glyphosate aos 20 dias após a emergência, época em que apresentavam quatro folhas verdadeiras. As subdoses de glyphosate, simulando deriva, foram de 270 e $540 \mathrm{~g} \mathrm{ha}^{-1}$. Também foi utilizada testemunha, sem aplicação do herbicida, para efeito de comparação. Foram realizadas avaliações semanais até 42 dias após a aplicação dos tratamentos (DAA), período em que também foi tomada a altura das plantas. Os sintomas visuais de intoxicação iniciaram-se aos 3 DAA, caracterizados pelo amarelecimento das pontas das folhas mais novas, seguido de murchamento do ápice das plantas. Na dose de $270 \mathrm{~g} \mathrm{ha}^{-1}$ esses sintomas foram de baixa intensidade, mas a $540 \mathrm{~g} \mathrm{ha}^{-1}$ causaram, na maioria dos casos, toxidez "preocupante" a "muito alta". Os cultivares BRS Facual e FM 986 mostraram-se os mais suscetíveis. A altura das plantas foi mais afetada quando se aplicou a menor dose de glyphosate. Houve recuperação de todos os cultivares tratados com $270 \mathrm{~g} \mathrm{ha}^{-1}$ de glyphosate até os 42 DAA. Quando tratados com $540 \mathrm{~g} \mathrm{ha}^{-1}$ de glyphosate, os cultivares Fabrika, Coodetec 407, BRS-Facual e ITA-90 foram mais sensiveis, apresentando redução de altura entre 84 e $90 \%$ aos 42 DAA. Os cultivares menos sensiveis na dose de $270 \mathrm{~g} \mathrm{ha}^{-1}$ de glyphosate não foram os mesmos para a dose de $540 \mathrm{~g} \mathrm{ha}^{-1}$.
\end{abstract}

Palavras-chave: Gossypium hirsutum, simulação, deriva, herbicida, fitotoxicidade.

\begin{abstract}
The response of nine cotton cultivars economically important in the state of Mato Grosso was evaluated in relation to the toxicity caused by reduced rates of glyphosate. The cotton cultivars used were Fabrika, Makina, ITA-90, FM 986, FM 966, Delta Opal, BRS Facual, Antares and Coodetec 407. The plants were cultivated in plastic pots filled with soil substratum and kept under greenhouse conditions. Glyphosate application was performed 20 days post emergence, at which time they presented four true leaves. Reduced rates of glyphosate, simulating drift, ranged from 270 and $540 \mathrm{~g}$. A control was also used, without herbicide application to compare the effects. Weekly evaluations were accomplished up to 42 days after application (DAA). During this period, plant height was also measured. Visual symptoms began 3 DAA, with the appearance of yellow spots at the tips of the youngest leaves, followed by withering at the apex of the plants. At the rate of $270 \mathrm{~g} \mathrm{ha}^{-1}$, these symptoms showed low intensity but at the rate of $540 \mathrm{~g} \mathrm{ha}^{-1}$ the symptoms caused "worrisome" to "very high" toxicity, in most of the cases. BRS Facual and FM 986 were the most susceptible. Plant height was the most affected when the lowest glyphosate dose was applied. All cultivars treated with $270 \mathrm{~g} \mathrm{ha} \mathrm{I}^{-1}$ glyphosate rate until $42 \mathrm{DAA}$ were recovered. Fabrika,

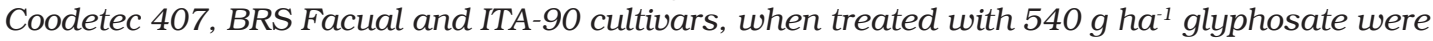
more sensitive, presenting a height reduction between 84 and 90\% 42 DAA. The less sensitive cultivars at the rate of $270 \mathrm{~g} \mathrm{ha}^{-1}$ glyphosate were not the same at the $540 \mathrm{~g} \mathrm{ha}^{-1}$ rate.
\end{abstract}

Key words: Gossypium hirsutum, simulation, drift, herbicide, phytotoxicity.

1 Recebido para publicação em 15/3/2005 e na forma revisada em 25/11/2005.

Parte da Dissertação de Mestrado do primeiro autor apresentada à FAMEV/UFMT - Cuiabá-MT

2 Eng.-Agrônomo, Professor do PCAA - Universidade do Estado de Mato Grosso, Caixa Postal 324, 78580-000 Alta FlorestaMT, <yama@ unemat.br>; ${ }^{3}$ Eng.-Agrônomo D.S., Professor da FAMEV - Universidade Federal de Mato Grosso, Av. Fernando Correa da Costa, s/n, 78100-000 Cuiabá-MT, <sheep@cpd.ufmt.br>. 


\section{INTRODUÇÃO}

No sistema de semeadura direta, o preparo mecânico do solo é substituído pela dessecação da vegetação, realizada por meio de herbicidas, dos quais o glyphosate é um dos mais empregados. Em razão do caráter não-seletivo desse herbicida, ele se torna tóxico para muitas plantas cultivadas, constituindo-se problema quando na ocorrência de deriva nas aplicações.

A cultura do algodoeiro vem ocupando posição de destaque no cenário agrícola de Mato Grosso (Gameiro et al., 2003), onde grande parte das áreas destinadas ao cultivo de grãos e fibras é realizada no sistema de semeadura direta e recebe aplicações do herbicida glyphosate em pré-plantio (Carvalho \& Chiavegato, 1999; Freire \& Farias, 2001). Derivas acidentais durante a aplicação têm potencial para provocar danos em cultivos no sistema de semeadura direta (Hemphill Jr. \& Montgomery, 1981; Gelmini, 1988; Ramsdale \& Messersmith, 2001; Banks \& Schroeder, 2002; Ellis et al., 2002; Lyon et al., 2003b) e no algodoeiro (Ellis \& Griffin, 2002; Lyon et al., 2003a; Miller et al., 2004), fazendo-se necessário gerar informações para a caracterização e quantificação desses efeitos. Também, a possibilidade de uso de cultivares de algodão, soja e milho geneticamente modificados para resistência ao herbicida glyphosate deverá aumentar o potencial de deriva desse produto para culturas/cultivares sensiveis.

Quando uma cultura apresenta problemas em seu desenvolvimento por suspeita de deriva de herbicidas, é necessário um diagnóstico, para o qual é muito importante conhecer os sintomas de intoxicação do produto na cultura e, também, fazer uma estimativa das perdas econômicas. Assim, a presente pesquisa foi realizada com o objetivo de estudar o efeito de subdoses de glyphosate, simulando deriva, em diferentes cultivares de algodoeiro de importância econômica para o Estado de Mato Grosso.

\section{MATERIAL E MÉTODOS}

O ensaio foi conduzido no período de setembro a novembro de 2002 , no viveiro da Escola Rural Produtiva, localizada nas coordenadas $10^{\circ} 04^{\prime} 59^{\prime \prime}$ latitude sul e $56^{\circ} 10^{\prime} 58^{\prime \prime}$ longitude oeste e a $302 \mathrm{~m}$ de altitude, no município de Alta Floresta - MT. O ambiente era protegido com tela para retenção de luminosidade, com valor nominal de $50 \%$, na parte superior e nas laterais.

Foi avaliado o efeito da aplicação de subdoses do herbicida glyphosate, simulando deriva, sobre o desenvolvimento inicial de nove cultivares de algodoeiro.

Foi usado o sal de isopropilamina de glyphosate, aplicado nas doses de 270 e $540 \mathrm{~g} \mathrm{ha}^{-1}$, sobre nove cultivares de algodoeiro, selecionados em função da importância atual e do potencial para o Estado de Mato Grosso: ITA 90, Antares, BRS-Facual, Delta opal, Fabrika, Makina, FM 966, FM 986 e Coodetec 407. As doses foram estabelecidas com base em ensaios preliminares. Também foi utilizada testemunha, sem aplicação do herbicida, para efeitos de comparação.

Foi adotado o delineamento experimental inteiramente casualizado, com três repetições, e os tratamentos foram dispostos num esquema fatorial $3 \times 9$. Cada unidade experimental foi representada por um vaso plástico com capacidade para $4 \mathrm{~L}$, preenchido com substrato de solo, proveniente de áreas de barranco. O substrato de solo foi peneirado e a fertilidade corrigida, procurando-se atingir os padrões exigidos pela cultura do algodoeiro (Pedroso Neto et al., 1999).

A semeadura foi feita a $3 \mathrm{~cm}$ de profundidade, colocando-se quatro sementes em cada vaso, realizando-se desbaste 15 dias após, deixando apenas uma planta por vaso. A irrigação foi realizada diariamente por meio de rega manual (regador), no período matutino, fornecendo água suficiente para que não houvesse déficit hídrico durante a condução do ensaio.

O herbicida foi aplicado quando as plantas estavam com quatro folhas verdadeiras (20 dias após a emergência das plântulas), por meio de um pulverizador costal pressurizado a $\mathrm{CO}_{2}$, mantido à pressão constante de $440 \mathrm{kPa}$, portando barra com um bico, munido de ponta Teejet de jato plano 110.02 , com gasto de calda de $100 \mathrm{~L} \mathrm{ha}^{-1}$. No momento da aplicação a temperatura ambiente era de $28^{\circ} \mathrm{C}$, a umidade relativa do ar de $75 \%$, o céu tinha $20 \%$ de nebulosidade e não havia ventos. Foi 
adicionado à calda do herbicida um espalhante adesivo contendo $200 \mathrm{~g} \mathrm{~L}^{-1}$ de nonil fenoxi poli (etilenoxi) etanol, na dose de 0,03\% v/v. Esse adjuvante foi acrescentado com o objetivo de potencializar os possiveis efeitos de intoxicação nas plantas de algodão dos diferentes cultivares.

\section{Características avaliadas}

a) Alterações visuais nas folhas - foram realizadas avaliações visuais de intoxicação nas plantas aos 7, 14, 21, 28, 35 e 42 dias após o tratamento, adotando-se a escala de notas apresentada na Tabela 1.

b) Altura de planta - esta característica foi tomada aos 7, 14, 21, 28, 35 e 42 dias após a aplicação do herbicida, medindo-se todas as plantas, da região do colo até a gema apical da haste principal. Os dados foram transformados em porcentual em relação à testemunha, para cada cultivar e época de avaliação.

Os dados coletados foram submetidos à análise de variância, comparando-se as médias das variáveis qualitativas, ou quantitativas com dois níveis, pelo teste de Scott \& Knott em nível de $10 \%$ de probabilidade.

\section{RESULTADOS E DISCUSSÃO}

\section{Alterações visuais na folha}

Os sintomas visuais de intoxicação nas folhas caracterizaram-se pela clorose do limbo foliar, mais rápida e intensa nas primeiras épocas avaliadas e na maior dose do glyphosate, que em alguns cultivares evoluiu para a morte. Além disso, algumas plantas, principalmente quando submetidas à menor dose, apresentaram superbrotamento.

As notas de intoxicação foram influenciadas pela dose de glyphosate em todas as avaliações $(\mathrm{p}<0,01)$ e pela interação entre cultivares e doses nas avaliações aos 14, 21, $28,35$ e 42 dias ( $<<0,05)$.

Nas Figuras 1 e 2 são apresentadas as notas de intoxicação dos nove cultivares de algodoeiro tratados com 270 e $540 \mathrm{~g} \mathrm{ha}^{-1}$ de glyphosate, aos 7, 14, 21, 28, 35 e 42 dias após a aplicação.

Na dose de $270 \mathrm{~g} \mathrm{ha}^{-1}$, durante todas as avaliações, os sintomas de intoxicação foram classificados como leves ou moderados, com sensivel evolução dos sintomas aos 14 DAA e, logo em seguida, posterior redução com a metabolização da molécula herbicida, não havendo, entretanto, diferença no comportamento entre os cultivares. Quando se aplicaram $270 \mathrm{~g} \mathrm{ha}^{-1}$ de glyphosate, apesar de não ter ocorrido aumento expressivo na intoxicação a partir dos 14 DAA, a recuperação dos sintomas foi lenta (Figura 1).

Quando tratados com $540 \mathrm{~g} \mathrm{ha}^{-1} \mathrm{de}$ glyphosate, os sintomas de intoxicação foram classificados como "preocupante" a "muito alta", e a resposta diferencial entre os cultivares se manifestou a partir dos 14 DAA, com os cultivares Fabrika, BRS-Facual, Delta opal e ITA-90 apresentando as maiores notas (97, 90, 87 e 93, respectivamente). Até o final das avaliações, os cultivares Fabrika e BRS-Facual mantiveram notas superiores a 93 .

Tabela 1 - Escala de notas utilizada para a avaliação visual de fitotoxicidade de herbicidas

\begin{tabular}{|c|c|c|}
\hline Conceito & Nota & Observação \\
\hline Muito leve & $0-5$ & $\begin{array}{l}\text { Sintomas fracos ou pouco evidentes. Nota zero quando não se observam quaisquer alterações } \\
\text { na cultura. }\end{array}$ \\
\hline Leve & $6-10$ & Sintomas nítidos, de baixa intensidade. \\
\hline Moderada & $11-20$ & Sintomas nítidos, mais intensos que na classe anterior. \\
\hline Aceitável & $21-35$ & Sintomas pronunciados, porém totalmente tolerados pela cultura. \\
\hline Preocupante & $36-45$ & $\begin{array}{l}\text { Sintomas mais drásticos que na categoria anterior, mas ainda passíveis de recuperação, e sem } \\
\text { expectativas de redução no rendimento econômico. }\end{array}$ \\
\hline Alta & $46-60$ & Danos irreversíveis, com previsão de redução no rendimento econômico. \\
\hline Muito alta & $61-100$ & $\begin{array}{l}\text { Danos irreversíveis muito severos, com previsão de redução drástica no rendimento econômico. } \\
\text { Nota } 100 \text { para morte de toda a cultura. }\end{array}$ \\
\hline
\end{tabular}

Adaptado de SBCPD (1995). 


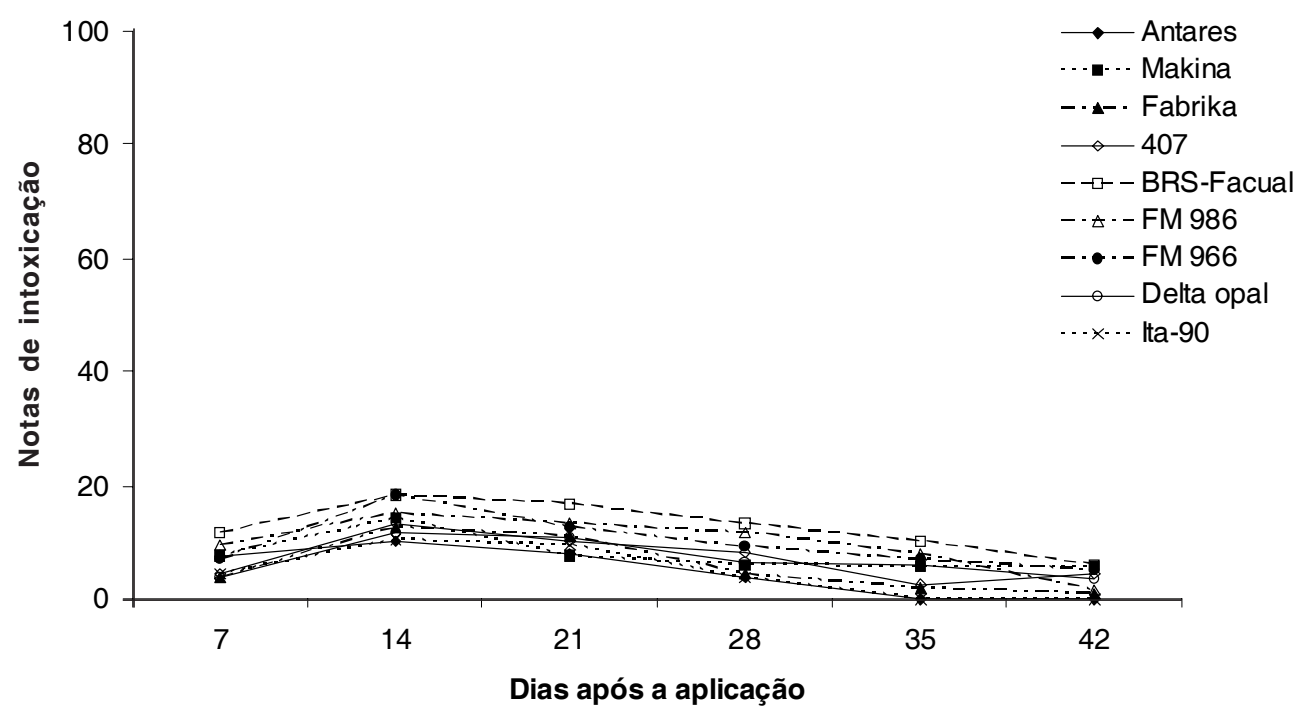

Figura 1 - Notas de intoxicação em nove cultivares de algodoeiro tratados com $270 \mathrm{~g} \mathrm{ha}^{-1}$ de glyphosate. Alta Floresta-MT. 2003.

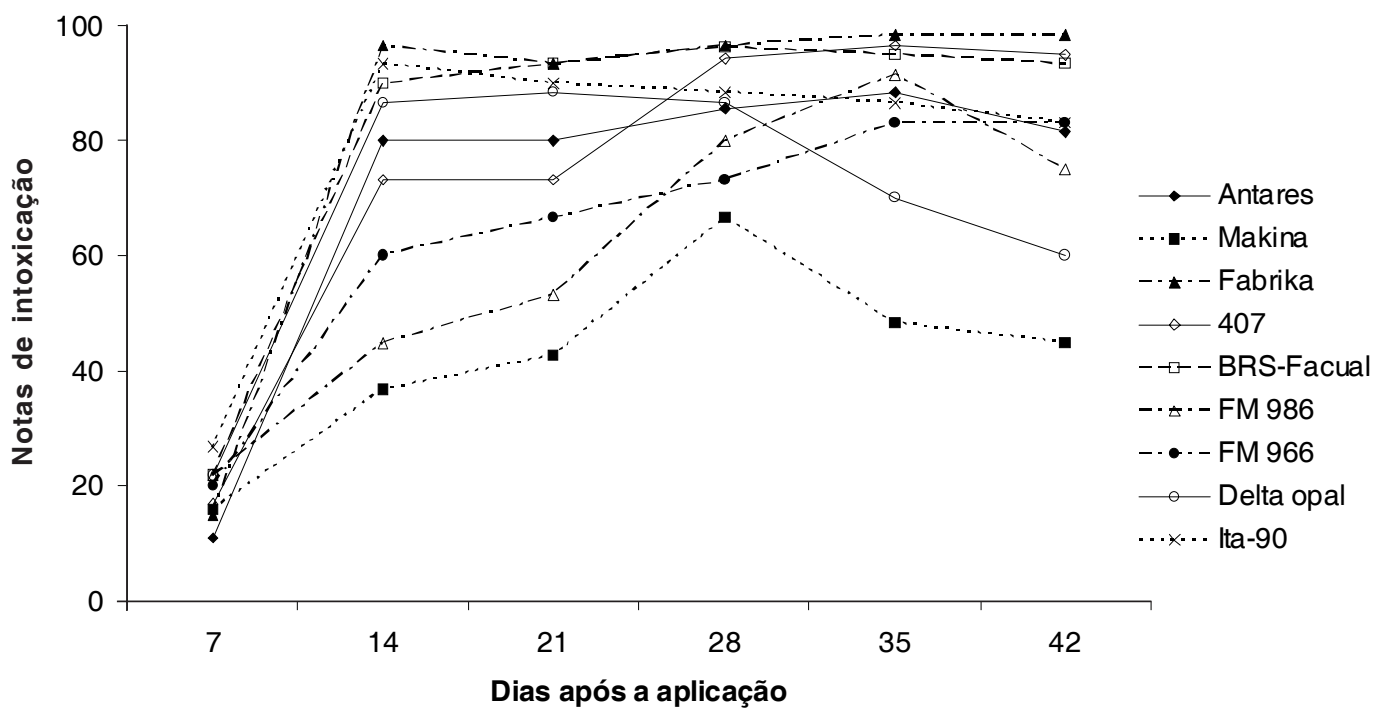

Figura 2 - Notas de intoxicação em nove cultivares de algodoeiro tratados com $540 \mathrm{~g} \mathrm{ha}^{-1}$ de glyphosate. Alta Floresta-MT. 2003.

Os cultivares Makina e FM-986 apresentaram evolução dos sintomas mais lenta que a dos demais cultivares até a avaliação aos 28 DAA. A partir daí, o cultivar Makina apresentou decréscimo nos sintomas de intoxicação, chegando à última avaliação com nota 45 (Figura 2).

Desde a primeira avaliação, a diferença entre as duas doses de glyphosate foi bem distinta, com notas mais altas para a dose mais elevada de glyphosate.
Os leves sintomas de intoxicação nas folhas do algodoeiro observados quando tratados com $270 \mathrm{~g} \mathrm{ha}^{-1}$ de glyphosate corroboram os obtidos por Ellis \& Griffin (2002) e Miller et al. (2004), que relataram a presença de intoxicação somente em doses superiores a $140 \mathrm{~g} \mathrm{ha}^{-1}$. Esse comportamento do algodoeiro em relação ao glyphosate difere do de outras culturas, como milho e sorgo, cujos sintomas são altos em doses inferiores a $172 \mathrm{~g} \mathrm{ha}^{-1}$ (Magalhães et al., 2001a, b). 
Os sintomas menos pronunciados obtidos com o cultivar Makina permitem inferir a diferença de comportamento entre cultivares em relação ao herbicida glyphosate, fato não observado por Ellis \& Griffin (2002), que, testando cinco cultivares, não encontraram diferença entre eles. Estudos futuros sobre a base da resposta diferencial de cultivares de algodoeiro ao glyphosate poderiam fornecer subsídios aos melhoristas interessados no desenvolvimento de cultivares com menor sensibilidade a esse herbicida.

\section{Altura de planta}

A altura de plantas foi influenciada pelo cultivar e pela dose $(p<0,10)$ em todas as avaliações e pela interação entre cultivar e dose $(\mathrm{p}<0,10)$ aos 14, 21, 28, 35 e 42 dias após a aplicação.

Na Tabela 2 são apresentadas as médias de altura de planta aos 14, 21, 28, 35 e 42 dias, em porcentagem em relação à testemunha, comparando, dentro de cada época de avaliação, o efeito de cultivar dentro de cada dose de

Tabela 2 - Médias de altura de cultivares de algodoeiro (\% em relação à testemunha) tratados com $270 \mathrm{e} 540 \mathrm{~g}$ ha $^{-1} \mathrm{de}$ glyphosate, aos 14, 21, 28, 35 e 42 dias após a aplicação. Alta Floresta-MT. 2003

\begin{tabular}{|c|c|c|c|c|c|c|c|c|c|c|}
\hline \multirow{3}{*}{ Cultivar } & \multicolumn{2}{|c|}{14 DAA } & \multicolumn{2}{|c|}{$21 \mathrm{DAA}$} & \multicolumn{2}{|c|}{$28 \mathrm{DAA}$} & \multicolumn{2}{|c|}{35 DAA } & \multicolumn{2}{|c|}{42 DAA } \\
\hline & \multicolumn{10}{|c|}{ Dose $\left(\mathrm{g} \mathrm{ha}^{-1}\right)$} \\
\hline & 270 & 540 & 270 & 540 & 270 & 540 & 270 & 540 & 270 & 540 \\
\hline Antares & $99 \mathrm{Aa}$ & $59 \mathrm{Ba}$ & $92 \mathrm{Aa}$ & $45 \mathrm{Ba}$ & $95 \mathrm{Aa}$ & $37 \mathrm{Ba}$ & $94 \mathrm{Aa}$ & $33 \mathrm{Ba}$ & $101 \mathrm{Aa}$ & $29 \mathrm{Ba}$ \\
\hline Makina & $74 \mathrm{Aa}$ & $61 \mathrm{Aa}$ & $67 \mathrm{Ab}$ & $52 \mathrm{Aa}$ & $75 \mathrm{Ab}$ & $47 \mathrm{Ba}$ & $79 \mathrm{Aa}$ & $51 \mathrm{Ba}$ & $81 \mathrm{Ab}$ & $55 \mathrm{Ba}$ \\
\hline Fabrika & $71 \mathrm{Aa}$ & $14 \mathrm{Bb}$ & $64 \mathrm{Ab}$ & $12 \mathrm{Bb}$ & $70 \mathrm{Ab}$ & $11 \mathrm{Bb}$ & $75 \mathrm{Aa}$ & $10 \mathrm{Bb}$ & $85 \mathrm{Ab}$ & $9 \mathrm{Bb}$ \\
\hline Coodetec 407 & $84 \mathrm{Aa}$ & $42 \mathrm{Ba}$ & $78 \mathrm{Aa}$ & $36 \mathrm{Ba}$ & $83 \mathrm{Aa}$ & $31 \mathrm{Ba}$ & $82 \mathrm{Aa}$ & $17 \mathrm{Bb}$ & $85 \mathrm{Ab}$ & $17 \mathrm{Bb}$ \\
\hline BRS-Facual & $68 \mathrm{Aa}$ & $48 \mathrm{Aa}$ & $58 \mathrm{Ab}$ & $14 \mathrm{Bb}$ & $59 \mathrm{Ab}$ & $13 \mathrm{Bb}$ & $63 \mathrm{Aa}$ & $12 \mathrm{Bb}$ & $61 \mathrm{Ab}$ & $13 \mathrm{Bb}$ \\
\hline FM-986 & $64 \mathrm{Aa}$ & $53 \mathrm{Aa}$ & $59 \mathrm{Ab}$ & $45 \mathrm{Aa}$ & $59 \mathrm{Ab}$ & $41 \mathrm{Aa}$ & $62 \mathrm{Aa}$ & $37 \mathrm{Ba}$ & $69 \mathrm{Ab}$ & $35 \mathrm{Ba}$ \\
\hline FM-966 & $80 \mathrm{Aa}$ & $70 \mathrm{Aa}$ & $78 \mathrm{Aa}$ & $61 \mathrm{Aa}$ & $86 \mathrm{Aa}$ & $56 \mathrm{Ba}$ & $93 \mathrm{Aa}$ & $51 \mathrm{Ba}$ & $103 \mathrm{Aa}$ & $48 \mathrm{Ba}$ \\
\hline Delta opal & $70 \mathrm{Aa}$ & $46 \mathrm{Ba}$ & $63 \mathrm{Ab}$ & $30 \mathrm{Bb}$ & $75 \mathrm{Ab}$ & $27 \mathrm{Bb}$ & $79 \mathrm{Aa}$ & $30 \mathrm{Ba}$ & $81 \mathrm{Ab}$ & $32 \mathrm{Ba}$ \\
\hline ITA-90 & $86 \mathrm{Aa}$ & $39 \mathrm{Ba}$ & $84 \mathrm{Aa}$ & $13 \mathrm{Bb}$ & $91 \mathrm{Aa}$ & $11 \mathrm{Bb}$ & $94 \mathrm{Aa}$ & $15 \mathrm{Bb}$ & $97 \mathrm{Aa}$ & $15 \mathrm{Bb}$ \\
\hline
\end{tabular}

Médias dentro de cada época de avaliação que tenham em sua sequiência pelo menos uma letra em comum, maiúsculas nas linhas e minúsculas nas colunas, não diferem entre si pelo teste de Scott \& Knott a 10\% de probabilidade.

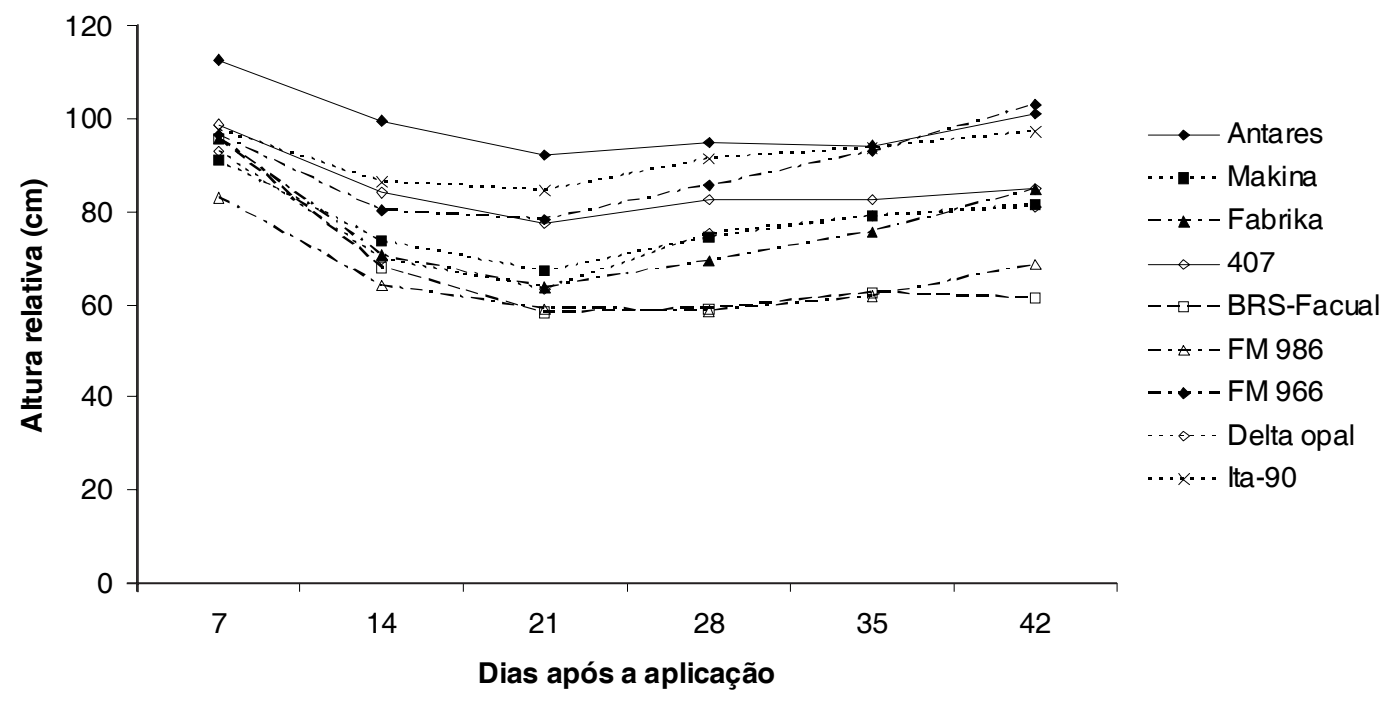

Figura 3 - Altura relativa de nove cultivares de algodoeiro (\% em relação à testemunha) tratados com $270 \mathrm{~g}^{-1}$ de glyphosate. Alta Floresta-MT. 2003. 


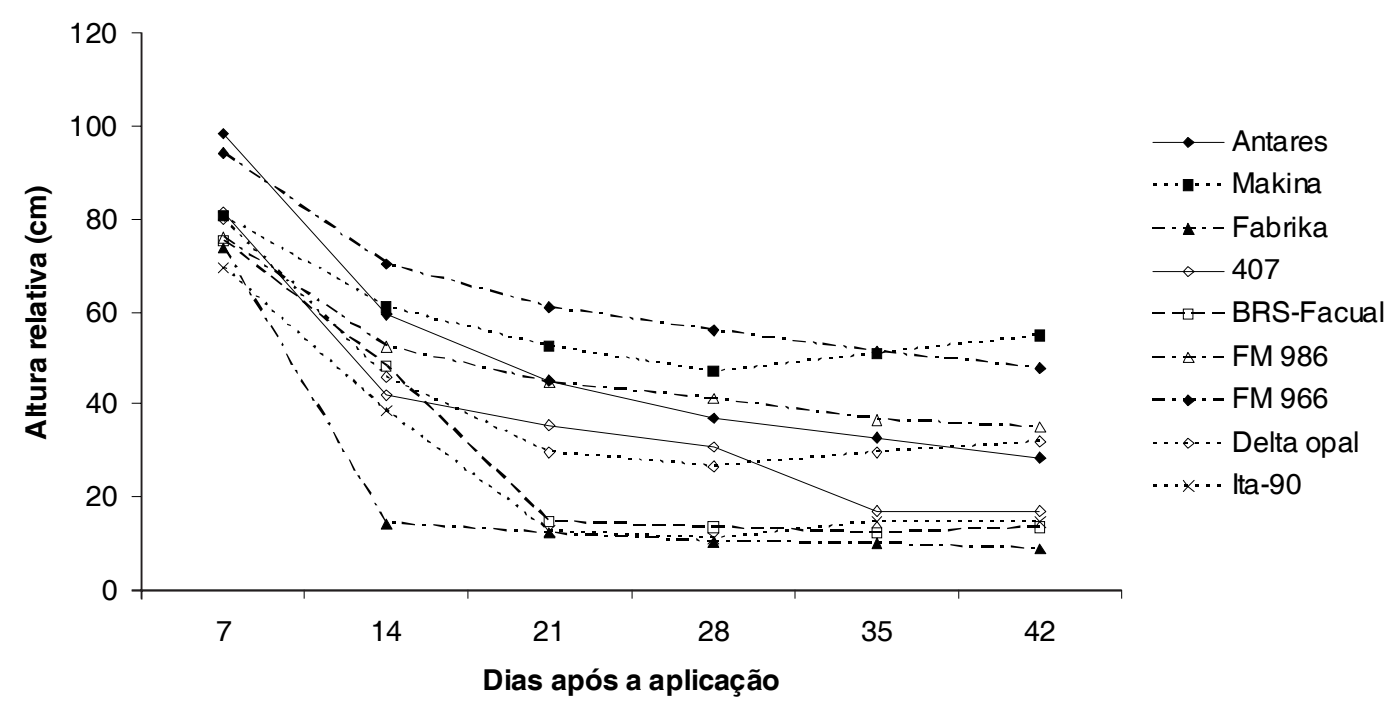

Figura 4 - Altura relativa de nove cultivares de algodoeiro (\% em relação à testemunha) tratados com $540 \mathrm{~g}^{-1} \mathrm{de}^{-1} \mathrm{glyphosate}$. Alta Floresta-MT. 2003.

glyphosate e o efeito de dose de glyphosate dentro de cada cultivar. O comportamento dessa característica, ao longo de todo o período de avaliação, é ilustrado nas Figuras 3 e 4.

Na dose de $270 \mathrm{~g} \mathrm{ha}^{-1}$, aos 14 DAA, os cultivares apresentaram retardo no crescimento entre 1 e 36\%, sem diferença significativa entre eles. Na avaliação seguinte (21 DAA), o retardo foi observado em todos os cultivares, com Makina, Fabrika, BRS-Facual, FM-986 e Delta opal apresentando diferenças significativas entre 33 e $42 \%$. Aos 28 DAA, diferença significativa de 25 e $41 \%$ foi observada nos mesmos cultivares. Aos 35 DAA, não houve diferença significativa entre os cultivares, com retardo médio entre 6 e 38\%.

Aos 42 DAA, à exceção dos cultivares Antares, FM-966 e ITA-90, os demais apresentaram retardo no crescimento entre 25 e $39 \%$.

Maiores retardos no crescimento ocorreram quando as plantas foram tratadas com a dose mais elevada (540 $\mathrm{g} \mathrm{ha}^{-1}$ ), com padrão diferenciado entre os cultivares. Aos 14 DAA, o cultivar Fabrika foi o mais sensível, apresentando retardo de $86 \%$. Retardos entre 70 e 89\% foram observados nas avaliações aos $21 \mathrm{e}$ 28 DAA, sendo mais sensiveis os cultivares Fabrika, BRS-Facual, Delta opal e ITA-90. À exceção do cultivar Delta opal, os mesmos cultivares e Coodetec 407 apresentaram retardos entre 83 e $90 \%$ aos 35 DAA. Na última avaliação, aos 42 DAA, os cultivares Fabrika, BRS-Facual, ITA-90 e Coodetec 407 mostraram retardos médios de altura de $91,87,85$ e $83 \%$, respectivamente, diferindo dos demais, que apresentaram retardos médios entre 45 e $71 \%$.

Os cultivares menos sensiveis na dose de $270 \mathrm{~g} \mathrm{ha}^{-1}$ de glyphosate não foram necessariamente os menos sensiveis para a dose de $540 \mathrm{~g} \mathrm{ha}^{-1}$, como o cultivar ITA-90, que apresentou recuperação de $97 \%$ na dose de $270 \mathrm{~g} \mathrm{ha}^{-1}$ de glyphosate, com retardo de $85 \%$ na maior dose.

Na menor dose, o retardo na altura relativa se tornou mais expressivo dos 14 aos 21 DAA, com pouca recuperação até os 42 DAA para todos os cultivares. Os cultivares FM-966 e Fabrika foram os que mostraram maiores recuperações, chegando aos 42 DAA com 24 e $21 \%$, respectivamente. Os demais cultivares apresentaram recuperações inferiores a $18 \%$.

Nos cultivares tratados com $540 \mathrm{~g} \mathrm{ha}^{-1}$, a redução na altura relativa foi mais drástica que na dose de $270 \mathrm{~g} \mathrm{ha}^{-1}$, com máximo ocorrendo aos 21 DAA para a maioria dos cultivares, não havendo recuperação até os 42 DAA. 


\section{LITERATURA CITADA}

BANKS, P. A.; SCHROEDER, J. Carrier volume affects herbicide activity in simulated spray drift studies. Weed Technol., v. 16, p. 833-837, 2002.

CARVALHO, L. H.; CHIAVEGATO, E. J. A cultura do algodão no Brasil: fatores que afetam a produtividade. In: CIA, E.; FREIRE, E. C.; SANTOS, W. J. (Eds.). Cultura do algodoeiro. Piracicaba: Potafós, 1999. p. 1-8.

ELLIS, J. M.; GRIFFIN, J. L. Soybean (Glycine max) and cotton (Gossypium hirsutum) response to simulated drift of glyphosate and glufosinate. Weed Technol., v. 16, p. 580-586, 2002.

ELLIS, J. M.; GRIFFIN, J. L.; JONES, C. A. Effect of carrier volume on corn (Zea mays) and Soybean (Glycine max) response to simulated drift of glyphosate and glufosinate.

Weed Technol., v. 16, p. 587-592, 2002.

FREIRE, E. C.; FARIAS, F. J. C. Cultivares de algodão para o Centro-Oeste. In: EMBRAPA AGROPECUÁRIA OESTE; Embrapa Algodão. Algodão: tecnologia de produção. Dourados: 2001. p. 159-180.

GAMEIRO, A. H.; MENEZES, S. M.; PEREZ, P. Algodão: queda de produção e alta de dólar elevam preços. Agrianual 2003. p. 193-207.

GELMINI, G. A. Herbicidas: indicações básicas. Campinas: Fundação Cargill, 1988. 334 p.

HEMPHILL Jr., D. D.; MONTGOMERY, M. L. Response of vegetable crops to sublethal application of 2,4 D. Weed Sci., v. 29, n. 6, p. 632-635, 1981.
LYON, L. L. et al. Non glyphosate tolerant cotton response to simulated drift rates of glyphosate. Proceedings...

Alabama: Southern Weed Science Society, 2003a. v. 56. p. 14.

LYON, L. L. et al. Sorghum response to simulated drift rates of glyphosate. Proceedings... Alabama: Southern Weed Science Society, 2003b. v. 56. p. 273.

MAGALHÃES, P. C. et al. Efeito de doses reduzidas de glyphosate e paraquat simulando deriva na cultura do milho. Planta Daninha, v. 19, n. 2, p. 247-253, 2001a.

MAGALHÃES, P. C. et al. Efeito de doses reduzidas de glyphosate e paraquat simulando deriva na cultura do sorgo. Planta Daninha, v. 19, n. 2, p. 255-262, 2001 b.

MILLER, D. K. et al. Response of non glyphosate resistant cotton to reduced rates of glyphosate. Weed Sci., v. 52, p. 178-182, 2004.

PEDROSO NETO, J. C. Algodão. In: RIBEIRO, A. C.; GUIMARÃES, P. T. G.; ALVAREZ, V. H. (Eds.). Recomendações para o uso de corretivos e fertilizantes em Minas Gerais - $\mathbf{5}^{\mathbf{a}}$ aproximação. Viçosa: Universidade Federal de Viçosa, 1999. p. 278-279.

RAMSDALE, B. K.; MESSERSMITH, C. G. Drift-reducing nozzle effects on herbicide performance. Weed Technol., v. 15. p. 453-460, 2001.

SOCIEDADE BRASILEIRA DA CIÊNCIA DAS PLANTAS DANINHAS - SBCPD. Procedimentos para instalação, avaliação e análise de experimentos com herbicidas. Londrina: 1995. 42 p. 